Tropical Journal of Pharmaceutical Research June 2018; 17 (6): 1135-1143

ISSN: $1596-5996$ (print); 1596-9827 (electronic)

(c) Pharmacotherapy Group, Faculty of Pharmacy, University of Benin, Benin City, 300001 Nigeria.

Available online at http://www.tjpr.org

Original Research Article

http://dx.doi.org/10.4314/tjpr.v17i6.21

\title{
Chemotherapy drug regimen optimization using deterministic oscillatory search algorithm
}

\author{
N Archana ${ }^{1 *}$, Antony Manoj Fh Benedict ${ }^{2}$, J Niresh ${ }^{3}$ \\ ${ }^{1}$ Department of Electrical \& Electronics Engineering, PSG College of Technology, Coimbatore 641004, India, ${ }^{2}$ Department of \\ Electrical and Computer Science, University of Rostock, Rostock, Germany, ${ }^{3}$ Department of Automobile Engineering, PSG \\ College of Technology, Coimbatore 641004, India
}

*For correspondence: Email: archana.nathan31@gmail.com

Sent for review: 12 February 2018

Revised accepted: 10 May 2018

\begin{abstract}
Purpose: To schedule chemotherapy drug delivery using Deterministic Oscillatory Search algorithm, keeping the toxicity level within permissible limits and reducing the number of tumor cells within a predefined time period.

Methods: A novel metaheuristic algorithm, deterministic oscillatory search, has been used to optimize the Gompertzian model of the drug regimen problem. The model is tested with fixed (fixed interval variable dose, FIVD) and variable (variable interval variable dose, VIVD) interval schemes and the dosage presented for 52 weeks. In the fixed interval, the treatment plan is fixed in such a way that doses are given on the first two days of every seven weeks such as day 7, day 14, etc.

Results: On comparing the two schemes, FIVD provided a higher reduction in the number of tumor cells by $98 \%$ compared to $87 \%$ by VIVD after the treatment period. Also, a significant reduction in the number was obtained half way through the regimen. The dose level and toxicity are also reduced in the FIVD scheme. The value of drug concentration is more in FIVD scheme (50) compared to VIVD (41); however, it is well within the acceptable limits of concentration. The results proved the effectiveness of the proposed technique in terms of reduced drug concentration, toxicity, tumor size and drug level within a predetermined time period.

Conclusion: Artificial intelligent techniques can be used as a tool to aid oncologists in the effective treatment of cancer through chemotherapy.
\end{abstract}

Keywords: Deterministic Oscillatory Search, Chemotherapy scheduling, Drug schedule, Artificial intelligence

This is an Open Access article that uses a funding model which does not charge readers or their institutions for access and distributed under the terms of the Creative Commons Attribution License (http://creativecommons.org/licenses/by/4.0) and the Budapest Open Access Initiative (http://www.budapestopenaccessinitiative.org/read), which permit unrestricted use, distribution, and reproduction in any medium, provided the original work is properly credited.

Tropical Journal of Pharmaceutical Research is indexed by Science Citation Index (SciSearch), Scopus, International Pharmaceutical Abstract, Chemical Abstracts, Embase, Index Copernicus, EBSCO, African Index Medicus, JournalSeek, Journal Citation Reports/Science Edition, Directory of Open Access Journals (DOAJ), African Journal Online, Bioline International, Open-J-Gate and Pharmacy Abstracts

\section{INTRODUCTION}

Cancer, considered as a fatal disease and a leading cause of mortality worldwide, is characterized by abnormal growth of cells, leading to the destruction of body tissue.
Cancers are generally treated with different procedures that include a combination of surgery, chemotherapy, radiation, hormone infusion, and immunotherapy. The selection of treatment depends on the type, stage and physical health of the patient. Chemotherapy is 
always considered as one of the most complex treatments, as it involves treating the patient with a combination of various drugs. Scheduling and choice of chemotherapy drugs are generally made with the help of information available in text books or journals with proven results for similar patients. In reality, effective drug combination and schedule are fixed for a specific cancer type, based on clinical trials.

The current strategy to schedule chemotherapy is based on the empirical values from the clinical trials conducted during the development of drugs. Clinical trials are enumeration based and hence incur huge cost and time as testing has to be done with many combinations. To overcome these limitations, the chemotherapy drug regimen can be modeled mathematically and solved using intelligent algorithms. This results in a low cost, quick optimized scheduling solution. The mathematical model of drug optimization is based on pharmacokinetic and pharmacodynamic process. Swan and Vincent [8] modeled the chemotherapy process as a mathematical optimization problem based on the Gompertzian model. An objective function based on toxicity and number of cells killed was developed by Zietz [9] to find the optimized schedule. Researchers have also worked on finding the optimal drug regimen based on the cell cycle [10,11]. Murray developed a new mathematical model based on drugs that are non-cell-cycle-specific $\quad[12,13]$. Other mathematical models based on compartment model [14], adjuvant chemotherapy [7], cellcycle-specific [15] and drug resistance [16] have also been developed to address the chemotherapy drug regimen problem. Each of these mathematical models is based on certain parameters of the chemotherapy procedure like toxicity, cells killed and cell cycle.

The mathematical models developed by various researchers can be solved using various methods ranging from numerical solutions to soft computing techniques. A numerical method was proposed by Martin to find an optimal schedule of chemotherapy drugs with the inclusion of constraints [17]. Pontryagin Maximum Principle [18], explicit formulation [8,9,13], Newton's method [19], iterative algorithms [20], nonlinear programming [21] are other mathematical techniques applied in the literature to solve the problem. Heuristic methods have also been lately applied to solve the chemotherapy problem. Distributed evolutionary computing [22], adaptive neural network [23], genetic algorithm [24], strength Pareto evolutionary algorithm [25], particle swarm optimization [26] and various hybrid approaches $[27,28]$ have been successfully applied to find an optimal drug regimen to treat cancer patients. In this paper, a new optimization algorithm, Deterministic Oscillatory Search (DOS) [29] has been applied to solve the Gompertzian chemotherapy drug regimen problem with an aim to reduce the number of tumor cells and maintain the toxicity levels within limits.

\section{METHODS}

\section{Problem statement}

A Gompertzian mathematical model [17] with intravenous drug infusion is considered in this paper. This model is based on the concept of slowing down of tumor cell growth with an increase in the size of the tumor cell and approaching a plateau population. The mathematical expression is characterized by the pharmacokinetics model of the drug as given in the differential equation (Eq 1).

$C(t)=D(t)-C(t)$

where $D(t)$ is the drug infusion and $C(t)$ is the plasma drug concentration. $\mathrm{C}(\mathrm{t})$ increases with increase in $\mathrm{D}(\mathrm{t})$ and then decreases as a firstorder elimination kinetics at a rate of $\lambda$, which is related to half-life with factor $\ln (2) / \lambda$. With the drug infused, the number of cancer cell changes as given in equation (2), which represents the Gompertzian cell fashion.

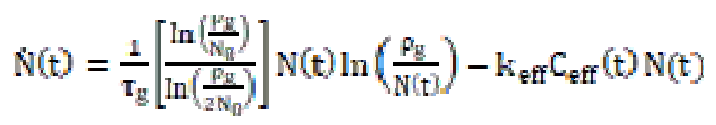

where $\mathrm{N}_{0}$ is the initial tumor population, $p_{\mathrm{g}}$ is the asymptotic plateau population, $\tau_{\mathrm{g}}$ is the doubling time of tumor during exponential growth. The first term in the equation represents the proliferation. The second term is the killer term which represents the death of the cell with $k_{\text {eff }}$ proportional to the number of tumor cells killed per unit per time per unit of drug. $\mathrm{C}_{\mathrm{eff}}$ is the drug concentration above the minimum therapeutic concentration $\left(C_{\mathrm{th}}\right)$ as expressed in equation (3).

$$
\begin{aligned}
& \mathrm{c}_{\mathrm{eff}}(\mathrm{t})=\left(\mathrm{c}(\mathrm{t})-\mathrm{C}_{\mathrm{th}}\right) \mathrm{H}\left(\mathrm{C}(\mathrm{t})-\mathrm{C}_{\mathrm{th}}\right) \ldots \ldots \ldots(3) \\
& H\left(C(t)-C_{t h}\right)=\left[\frac{1, \text { if } C\left(t_{j}\right) C_{t h}}{0, \text { if }}\right.
\end{aligned}
$$

The toxicity parameter is taken as in equation (5), where $\eta$ is a constant [30].

$\mathrm{T}(\mathrm{t})=\mathrm{C}(\mathrm{t})-\mathrm{n} \mathrm{T}(\mathrm{t})$ 
Based on the literature, three toxicity constraints and one efficacy constraint is considered in the proposed model [17,30,31] and given in equations (6)-(9).

$\mathrm{C}(\mathrm{t}) \leq \mathrm{C}_{\mathrm{max}}: \forall \mathrm{t} \in\left[0, \mathrm{t}_{\mathrm{f}}\right]$

$\mathbf{T}(\mathrm{t}) \leq \mathrm{T}_{\max }: \forall \mathrm{t} \in\left[0, \mathrm{t}_{\mathrm{f}}\right]$

$\int_{0}^{t_{T}} \mathrm{c}(\mathrm{t}) \mathrm{dt} \leq \mathrm{C}_{\mathrm{cum}}$

$N(t) \leq N_{0}: \forall t \in\left[0, t_{1}\right]$

$\mathrm{C}_{\max }, \mathrm{T}_{\max }$ and $\mathrm{C}_{\mathrm{cum}}$ are the maximum values of tolerable drug concentration, tolerable toxicity and drug exposure in plasma. Based on these expressions the objective function is designed to minimize the number of tumor cells at a finite time, which is taken as 52 weeks in the problem under study.

Minimize $e_{[(1)} N\left(t_{0}\right)$ satisfying the equations (1) to (9).

A novel meta heuristic algorithm used to solve the aforementioned objective function has been discussed in the next section.

\section{Deterministic oscillatory search}

Deterministic Oscillatory Search algorithm is not based on the natural phenomenon and hence has not been inspired by nature, like other meta heuristic algorithms. Gradient methods are capable of converging to a local optimum unless when the problem has noisy parameters with numerous peaks and valleys. Meta-heuristic algorithms are capable of solving this problem by using random numbers and the concept of swarming. Metaheuristic algorithms broadcast information obtained by each particle so that the entire community becomes fitter. Velocity based algorithms such as PSO, Firefly Algorithm, Symbiotic Organism Search, Gravitational Search Algorithm use it more directly when compared to evolution based algorithms such as Genetic Algorithm and Differential Evolution. As random numbers in an algorithm make it unpredictable in nature, DOS is an algorithm that has been developed with an inspiration to be a gradient based algorithm with the addition of swarming concept. The algorithm has also been developed with the motivation to create a robust technique that shows zero deviation when run several times with the same parameter settings. Since DOS algorithm does not incorporate random numbers, it provides same results for a given problem for any number of trials, making it extremely robust. The convergence characteristics are also found to be the same for each trial run. DOS can thus be summarized as an algorithm inspired to retain the robustness of classical methods with improved convergence characteristics as that of meta heuristic algorithms.

Deterministic Oscillatory Search algorithm consists of two components namely Initialization and Movement of particles. A combination based distribution is used in DOS algorithm where the particles are always influenced by attractive forces towards the global best and hence they fly towards the center. In the proposed algorithm, the particles are considered to move during each iteration under the influence of velocity. The velocity of the particle is initialized using Equation (10).

$v_{i}^{(0)}=\left[\frac{x_{\text {best }}-x_{i}^{(i)}}{2}\right]$

where $y_{i}^{0}$ and $x_{i}^{0}$ are the initial velocity and position of a particle 'i'. The particles move further based on gradient and swarming concept. In gradient based movement, the particles move along the direction which produces better results in consecutive trials. The velocity of the particle is halved and the direction is reversed if the fitness does not improve. The particle thus achieves the local optimum solution. This type of movement of the particle is similar to an oscillation or zig zag movement. A concept of fitness slope is incorporated in the algorithm, with three scenarios:

- Positive (current movement is producing better results)

- Negative (current movement is producing worse results)

- Unknown (velocity updated and slope is unknown)

The drawback of gradient technique when the search space has numerous peaks and valleys is overcome in DOS by incorporating swarming behaviour. Swarming is applied only when the particle fails to produce better results using gradient technique. The particle is made to move towards the global best position by updating the velocity using Equation (11).

$\mathrm{v}_{i}^{\mathrm{t}+1}=\mathrm{v}_{\mathrm{t}}^{\mathrm{t}}+\left[\frac{\mathrm{X}_{\mathrm{busit}}-\mathrm{x}_{\mathrm{i}}^{\mathrm{t}}}{2}\right]$

If the movement produces better results, the particle continues to find another local optimal solution and this process continues until the 
maximum number of evaluations is reached. The procedure to apply DOS algorithm to the chemotherapy optimization problem under study is briefed in the following pseudo code [29]:

Step 1: The number of particles and maximum number of iterations are initialized:

Step 2: The particles are initially placed in the search space using the combinatorial deterministic method

Step 3: For each particle, the fitness value is calculated and the best particle is identified

Step 4: The velocity of the particles are initialized using equation (1) and their fitness slope is initially set as unknown

Step 5: Iterative process while (number of eval < max eval) for ( $i=1$ :number of particles)

1. Calculate new position by adding velocity to the current position

2. Calculate the new fitness of the particle

3. Increment eval counter and update the Global best value

4. if (slope $==$ unknown)

Slope of the particle is updated to be either positive or negative based on the new fitness value

else if (slope $==$ positive)

if (new fitness is worse than old fitness)

(i) Update velocity $\rightarrow$ (-velocity/2)

(ii) Update slope to be negative

else if (slope $==$ negative)

Table 1: Parameter initialization if (new fitness is worse than the old fitness)

(i) Update velocity using Equation (2)

(ii) Update slope to be unknown

end if

end for

end while

Step 6: The position and fitness values of the global best particle are displayed.

\section{RESULTS}

The chemotherapy optimization problem is solved using the proposed DOS algorithm. The characteristics of DOS include zero randomness and no tunable parameter. Two schemes of drug scheduling: Fixed Interval Variable Dosage and Variable Interval Variable Dosage are implemented and the results are presented for 52 weeks. The number of iterations is taken as 30 , with the population size as 30 at an optimal level. The other parameters are set with the values as given in table 1. In the table, [D] represents the units of drug concentration/mass of drug delivered.

\section{FIVD scheme}

The fixed interval variable dose scheme involves treatment where the interval between consecutive dosages is kept as constant throughout the treatment cycle. The treatment plan is fixed in such a way that doses are given on the first two days of every seven weeks such as day 7, day 14 etc. Throughout the treatment plan, doses are given only 7 times. At the start of the treatment, a fixed high dose level of 50 [D] is administered.

\begin{tabular}{lcc}
\hline Parameter & Value & Unit \\
\hline $\mathrm{Tg}$ - First doubling time of the tumor during exponential growth & 150 & Days \\
$\mathrm{Q}_{\mathrm{g}}$ - Plateau population of cancer cells without treatment & $10^{12}$ & Cells \\
$\mathrm{N}_{0}$ - Initial cancer cell population & $10^{10}$ & Cells \\
$\mathrm{K}_{\text {eff }}$ - Fractional cell kill term for a highly effective drug & $2.7 \times 10^{-2}$ & $1 /$ days.[D] \\
$\lambda$ - Decrease in concentration of drug per unit time & 0.27 & $1 /$ days \\
$\mathrm{H}$ - Toxicity rate constant & 0.4 & $1 /$ days \\
$\mathrm{C}_{\text {th }}$ - Threshold drug concentration in plasma & 10 & [D] \\
$\mathrm{C}_{\text {max }}$ - Maximum tolerable drug concentration & 50 & [D] \\
$\mathrm{C}_{\text {cum }- \text { Maximum tolerable total drug exposure in plasma }}$ & $4.1 \times 10^{3}$ & [D].days \\
$\mathrm{T}_{\text {max }}$ Maximum tolerable toxicity & 100 & [D] \\
$\mathrm{T}_{\mathrm{f}}$ - Duration of treatment schedule & 364 & Days \\
\hline
\end{tabular}




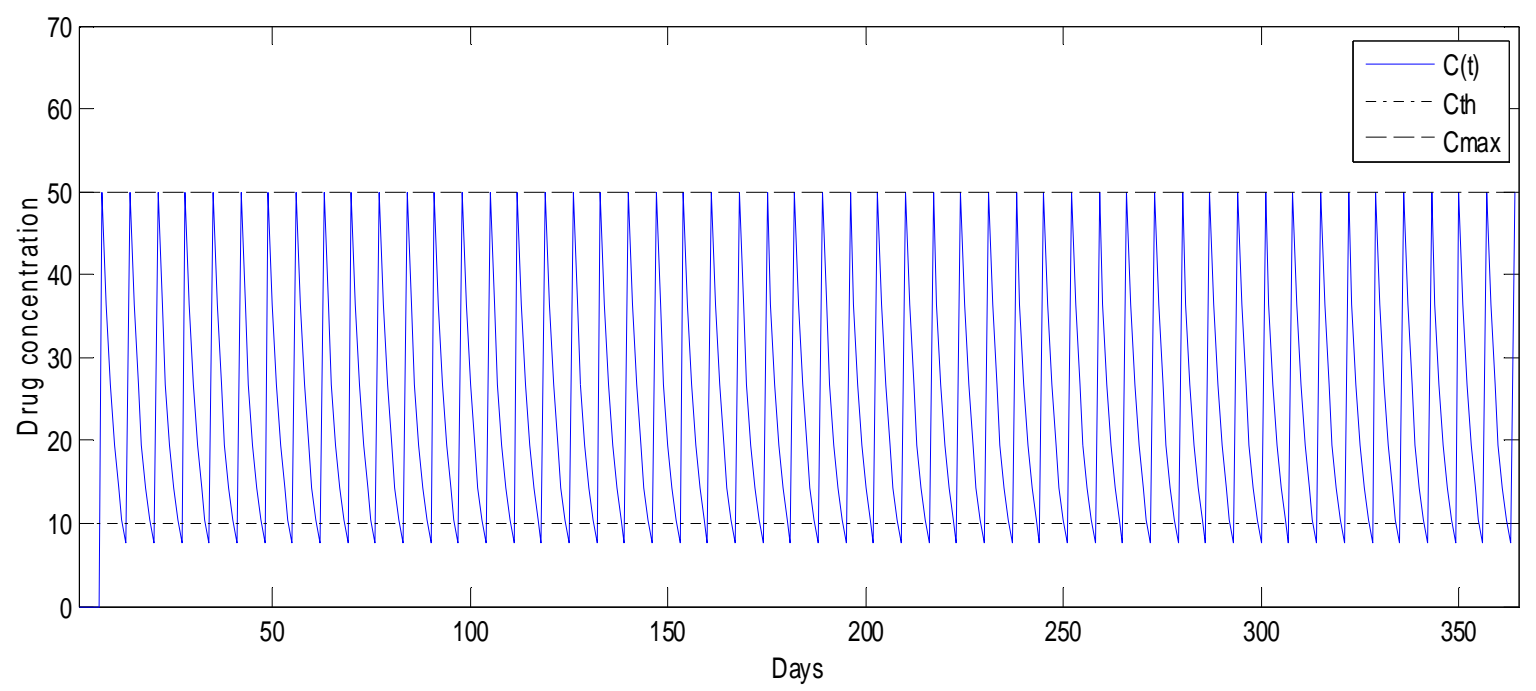

Figure 1: Drug concentration in FIVD scheme

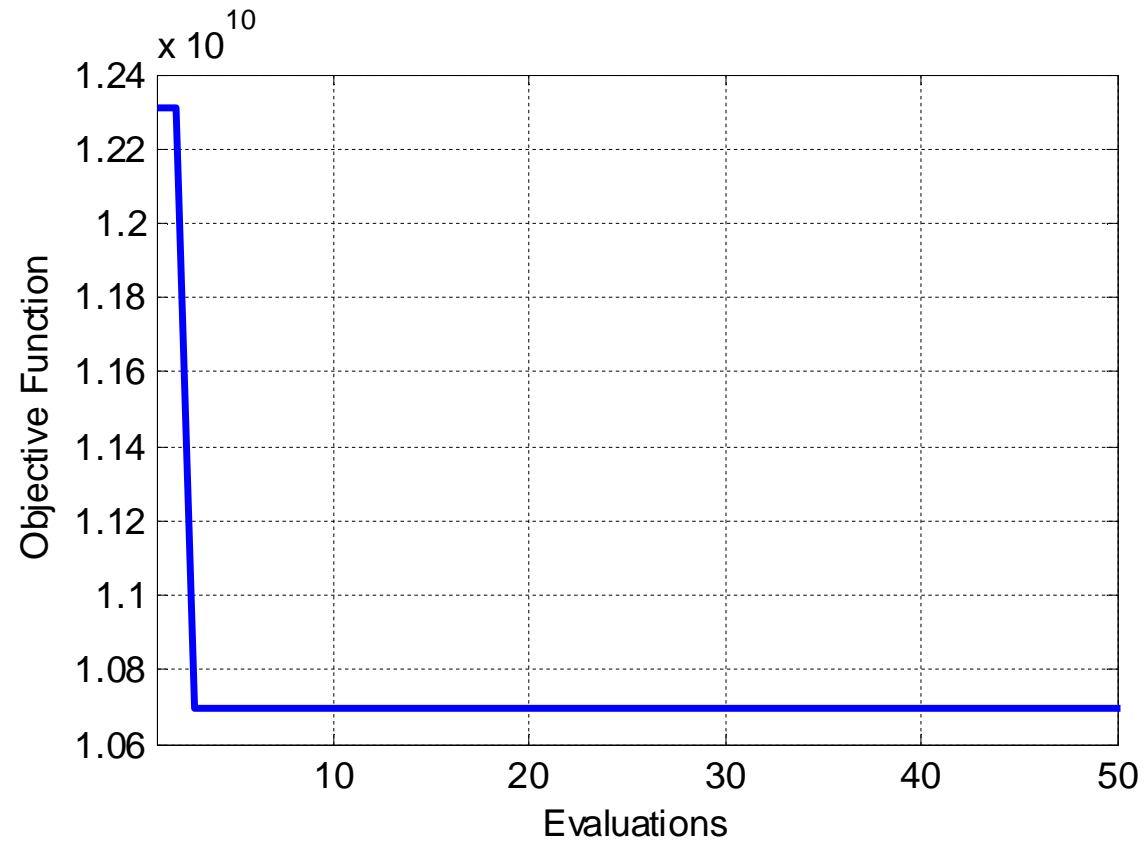

Figure 2: Convergence of DOS in FIVD scheme

The drug concentration in the FIVD scheme for 52 weeks is presented in Figure 1. The concentration of the drug reaches a maximum when it is administered, and then decreases until the next dosage after 7 weeks.

The convergence characteristics of DOS is presented in Figure 2 and it is clear that the DOS algorithm finds the solution in a minimum number of iterations. Also, the convergence characteristic of DOS does not change for any number of iterations. The reduction in the tumor cell number is evident from Figure 3 . The number of tumor cells decreases with every week's dosage and almost reaches to zero in a year's treatment schedule.
Figure 4 displays the toxicity level of the cells during the entire course of the drug regimen. It can be noted that the toxicity level is maintained well within the maximum limit during the chemotherapy treatment. The profile indicates that the toxicity increases when the drug is administered and decreases during the rest period.

\section{VIVD scheme}

Variable Interval Variable Dosage scheme includes drug regimen at unequal intervals of time. The rest period between successive dosages is also not equal. The number of iterations for this scheme is fixed as 50 and other 


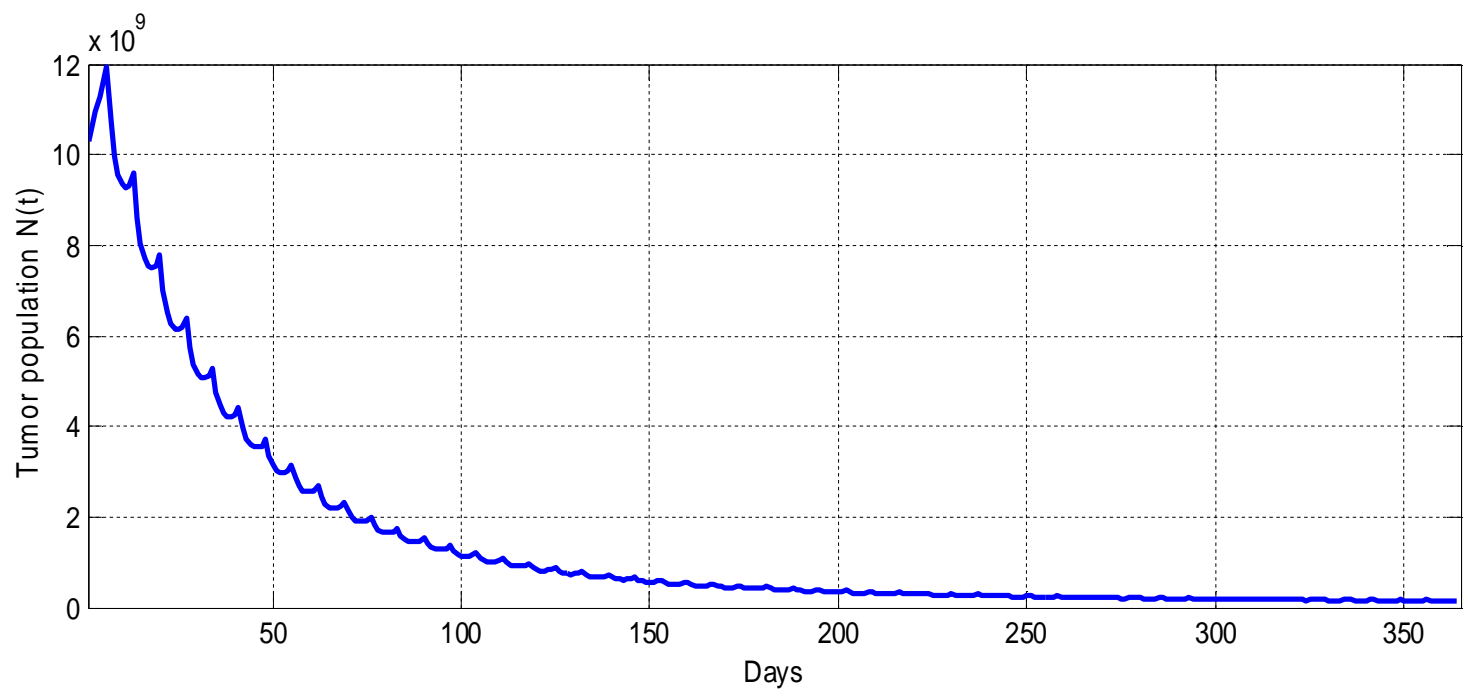

Figure 3: Tumor population in FIVD scheme

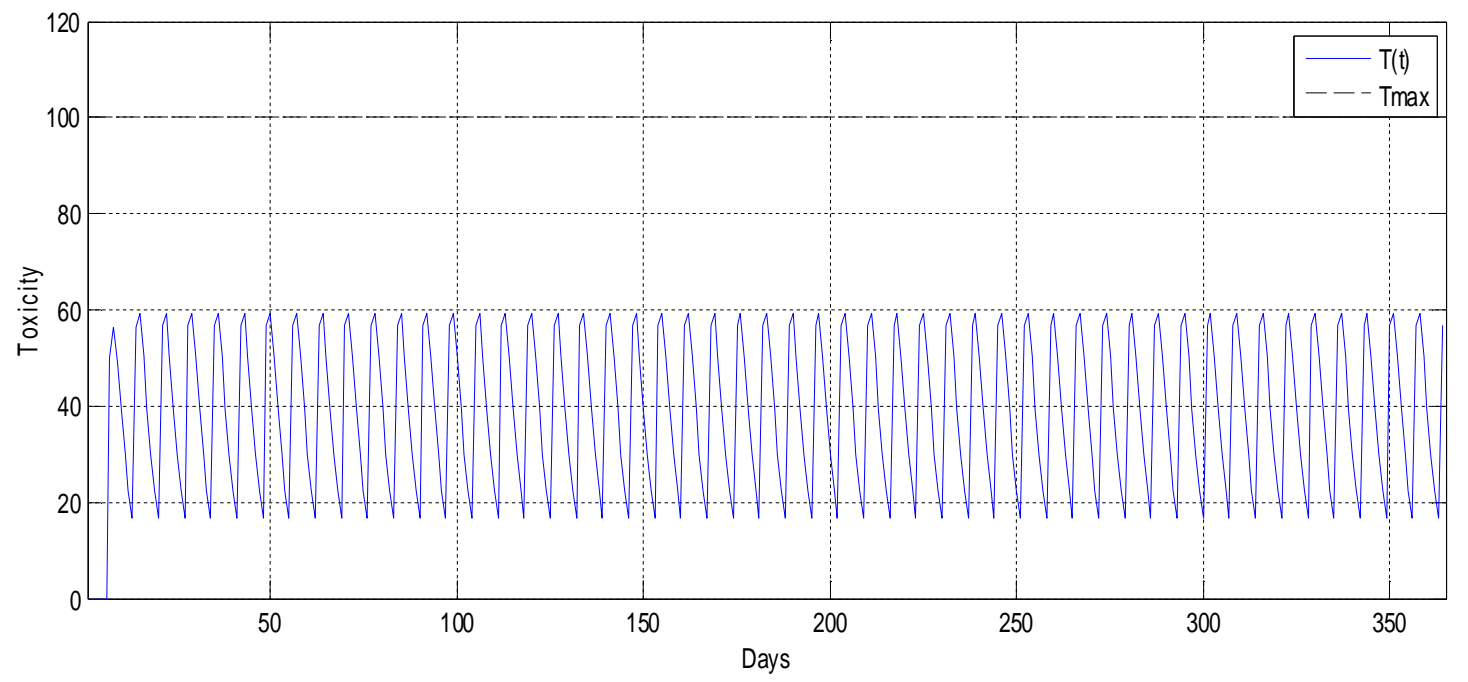

Figure 4: Toxicity profile in FIVD scheme

parameters are set as in Table 1. Figure 5 shows the concentration of the drug, which is maintained within the maximum limit of 50 . During the period when the drug is not administered for a long duration, the drug concentration goes down to zero and then again the dosage is scheduled for the patient. The convergence characteristic is given in Figure 6.

The population of the tumor cells, as seen from figure 7 , reaches approximately zero after the one-year drug regimen. It is seen that the population increases slightly during the long rest period. This is the limitation of VIVD scheme. Figure 8 displays the toxicity of the cells in the VIVD scheme, which is maintained below the maximum threshold of 100 .

The value of drug concentration is more in the FIVD scheme compared to VIVD; however, it is well within the acceptable limits of concentration. During the whole period of treatment for both schemes, the constraints mentioned in the previous sections have been considered and hence the toxicity, as well as drug concentration levels, do not cross the permissible limits. The drug schedule and rest period are carefully chosen so that the cancer cells do not aggravate or toxicity does not increase beyond the limit.

An analysis of the numerical results obtained during the VIVD and FIVD scheme is summarized in Table 2.

On comparing the two schemes, FIVD provides a better reduction in the number of tumor cells after the treatment period. Also, a significant reduction in the number is obtained half way through the regimen. The dose level and toxicity are also lower in the FIVD scheme.

\section{DISCUSSION}

Analysis of the results reveals that the determi- 


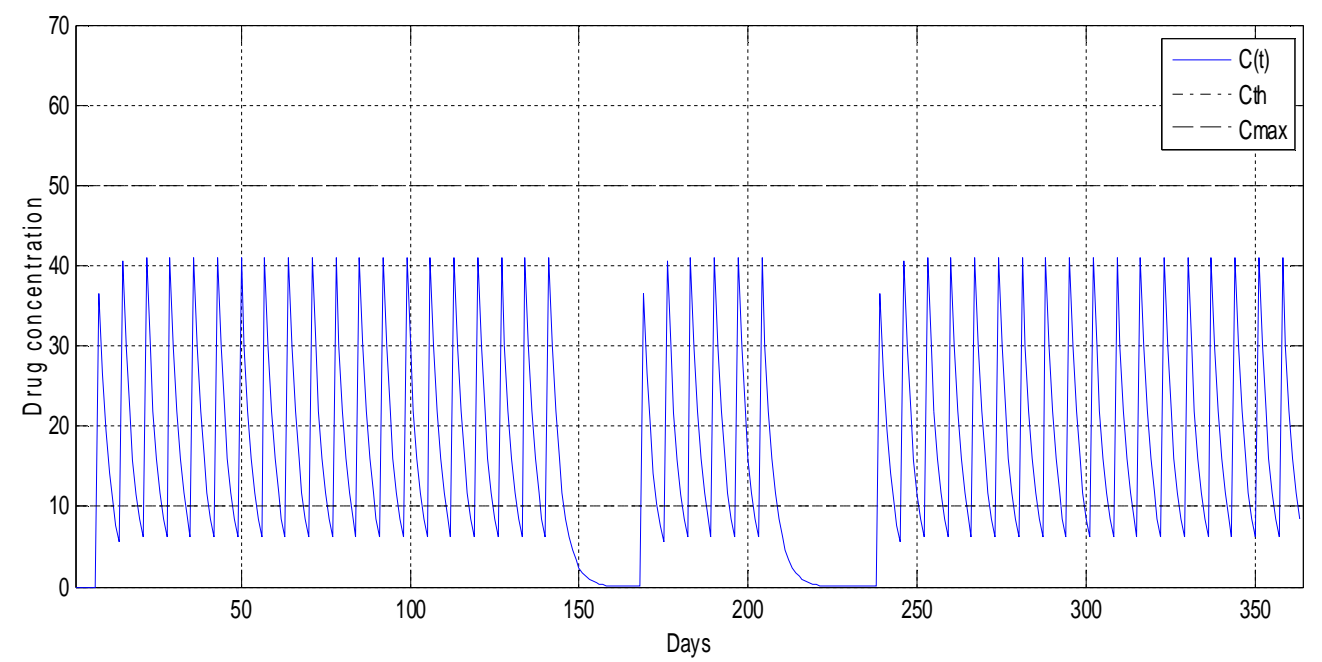

Figure 5: Drug concentration in VIVD scheme

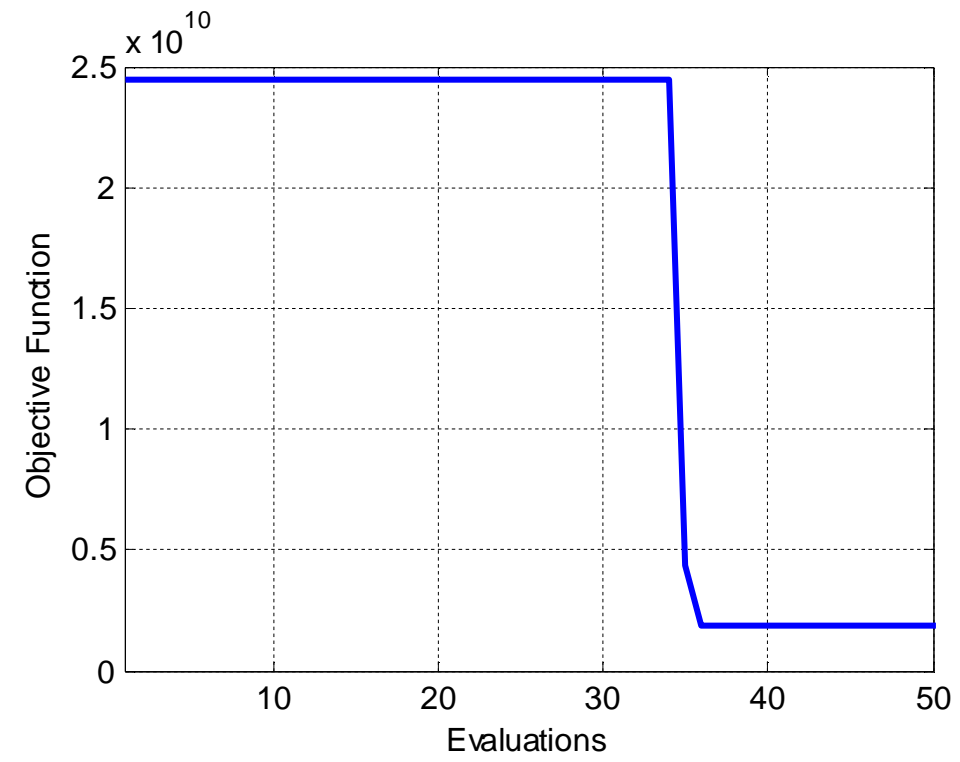

Figure 6: Convergence of DOS in VIVD scheme

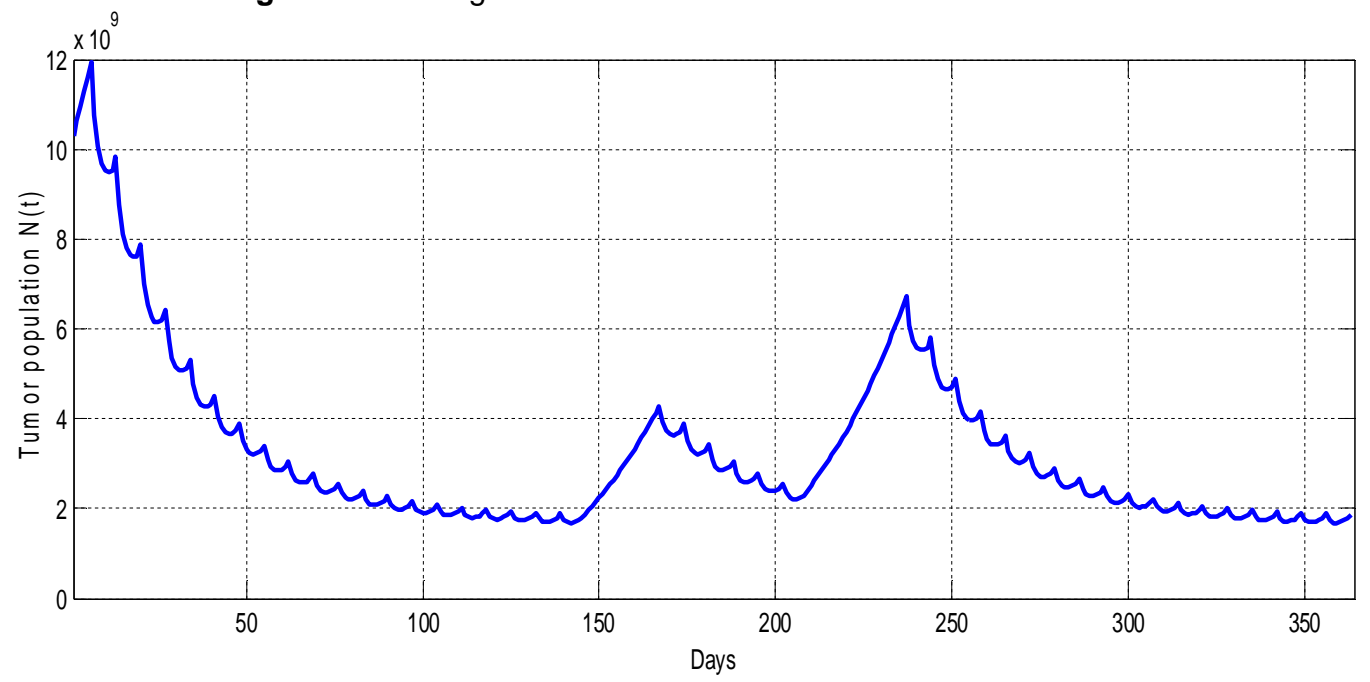

Figure 7: Tumor population in VIVD scheme 


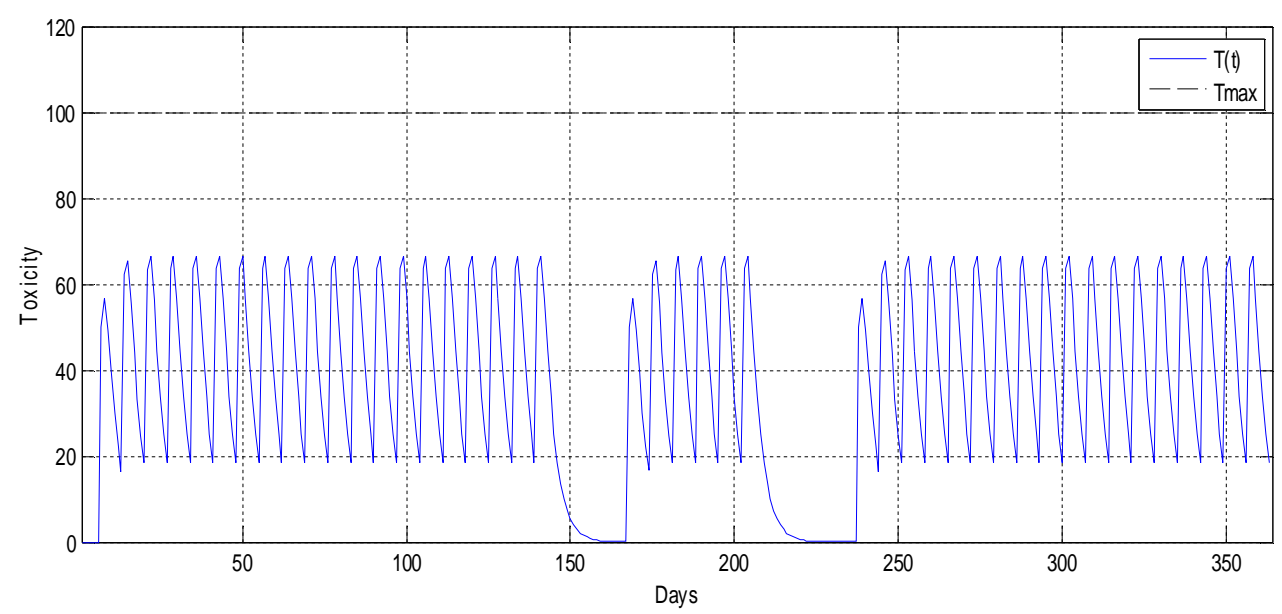

Figure 8: Toxicity profile in FIVD scheme

Table 2: Analysis of FIVD and VIVD

\begin{tabular}{lcc}
\hline Parameter & FIVD & VIVD \\
\hline Maximum drug dose [D] & 32.47 & 50 \\
Average drug dose [D] & 10 & 12 \\
Maximum drug concentration [D] & 50 & 41.03 \\
Average drug concentration [D] & 23.22 & 16.32 \\
Maximum toxicity [D] & 59.14 & 66.49 \\
Average toxicity [D] & 38.59 & 37.27 \\
Cell reduction percentage (\%) & 98 & 87 \\
\hline
\end{tabular}

nistic oscillatory search algorithm is able to solve the chemotherapy optimization problem successfully. Since DOS has gradient characteristics in addition to swarm intelligence, it performs better than the algorithms that rely on swarming behaviour alone. Another advantage of DOS is that the algorithm has to be evaluated only once to obtain the solution, in contrast to other algorithms, which are evaluated for many numbers of trials and the mean value is taken as the optimized solution. It can also solve problems irrespective of the number of global minima in the solution.

\section{CONCLUSION}

A new computationally feasible, fast, problemindependent soft computing algorithm has been applied to solve the chemotherapy drug scheduling problem. The core idea of this algorithm was inspired from the limitations observed from various existing metaheuristic algorithms like stochasticity, tunable parameters, and search behaviour. DOS algorithm has been developed with important features such as using no random numbers, single tuning parameter and search using gradient based technique inculcated with swarming nature. It is evident from the results that the FIVD scheme of treatment can cure the patients better than the VIVD scheme. The FIVD scheme results in a $98 \%$ reduction in tumor cells as compared to
$87 \%$ by VIVD. The maximum toxicity level is also less (59) compared to VIVD (66). The characteristics of the treatment such as regularity and less toxicity lead to better clinical feasibility.

\section{DECLARATIONS}

\section{Conflict of Interest}

No conflict of interest associated with this work.

\section{Contribution of Authors}

The authors declare that this work was done by the authors named in this article and all liabilities pertaining to claims relating to the content of this article will be borne by them.

\section{REFERENCES}

1. Andre $T$, Colin $P$, Louvet $C$, Gamelin E, Bouche $O$, Achille E, Colbert N, Boaziz C, Piedbois $P$, TubianaMathieu $N$ et al. Semimonthly Versus Monthly Regimen of Fluorouracil and Leucovorin Administered for 24 or 36 Weeks as Adjuvant Therapy in Stage II and III Colon Cancer: Results of a Randomized Trial. J Clin Oncol. 2003; 21: 2896-2903.

2. Thierry $A$, Corrado $B$, Lamia M-B, Matilde $N$, Josep $T$, Tamas H, Clare T, Marta Z, Philip C, et al. Oxaliplatin, Fluorouracil, and Leucovorin as Adjuvant Treatment for Colon Cancer. N Engl J Med. 2004; 350: 2343-2351

3. de Gramont A, Figer A, Seymour M, Homerin M, Hmissi $A$, Cassidy J, Boni C, Cortes-Funes $H$, Cervantes $A$, Freyer G. Leucovorin and Fluorouracil With or Without Oxaliplatin as First-Line Treatment in Advanced Colorectal Cancer. J Clin Oncol. 2000; 18: 2938-2947.

4. Francini G, Petrioli R, Lorenzini L, Mancini S, Armenio S, Tanzini G, Marsili S, Aquino A, Marzocca G, Civitelli S. Folinic acid and 5-fluorouracil as adjuvant chemotherapy in colon cancer. Gastroenterology. 1994; 106: 899-906. 
5. Wolmark N, Smith R, Fisher B, Bryant J, Hyams D, Atkins $J$, Dimitrov N, Oishi R, Prager $D$, Fehrenbacher $L$ et al. Adjuvant 5-Fluorouracil and Leucovorin With or Without Interferon Alfa-2a in Colon Carcinoma: National Surgical Adjuvant Breast and Bowel Project Protocol C-05. JNCI J Natl Cancer Inst. 1998; 90: 1810-1816.

6. de Gramont A, Bosset JF, Milan C, Rougier P, Bouche O, Etienne PL, Morvan F, Louvet C, Guillot T, Francois E et al. Randomized trial comparing monthly low-dose leucovorin and fluorouracil bolus with bimonthly highdose leucovorin and fluorouracil bolus plus continuous infusion for advanced colorectal cancer: a French intergroup study. J Clin Oncol. 1997; 15: 808-815.

7. Mamounas E, Wieand S, Wolmark N, Bear HD, Atkins $J N$, Song $K$, Jones J, Rockette $H$. Comparative efficacy of adjuvant chemotherapy in patients with Dukes' B versus Dukes' $C$ colon cancer: results from four National Surgical Adjuvant Breast and Bowel Project adjuvant studies (C-01, C-02, C-03, and C-04). J Clin Oncol. 1999; 17: 1349-1355.

8. Swan GW, Vincent TL. Optimal control analysis in the chemotherapy of $\lg G$ multiple myeloma. Bull Math Biol. 1977; 39: 317-337.

9. Zietz S, Nicolini C. Mathematical approaches to optimization of cancer chemotherapy. Bull Math Biol. 1979; 41: 305-324.

10. Dibrov BF, Zhabotinsky AM, Neyfakh YA, Orlova MP, Churikova LI. Mathematical model of cancer chemotherapy, periodic schedules of phase-specific cytotoxic-agent administration increasing the selectivity of therapy. Math Biosci. 1985; 73: 1-31.

11. Shin KG, Pado R. Design of optimal cancer chemotherapy using a continuous-time state model of cell kinetics. Math Biosci. 1982; 59: 225-248.

12. Murray JM. Optimal control for a cancer chemotherapy problem with general growth and loss functions. Math Biosci. 1990; 98: 273-287.

13. Murray JM. Some optimal control problems in cancer chemotherapy with a toxicity limit. Math Biosci. 1990; 100: 49-67.

14. Swierniak A, Smieja J. Analysis and optimization of drug resistant and phase-specific cancer chemotherapy models. Math Biosci Eng. 2005; 2: 657-670.

15. Swierniak A, Polanski A, Kimmel M. Optimal control problems arising in cell-cycle-specific cancer chemotherapy. Cell Prolif. 1996; 29: 117-139.

16. Swierniak A, Polanski A, Smieja J, Kimmel M. Modelling growth of drug resistant cancer populations as the system with positive feedback. Math Comput Model. 2003; 37: 1245-1252.
17. Martin RB. Optimal control drug scheduling of cancer chemotherapy. Automatica. 1992; 28: 1113-1123.

18. Panetta JC. A mathematical model of drug resistance: heterogeneous tumors. Math Biosci. 1998; 147: 41-61.

19. Martin RB, Fisher ME, Minchin RF, Teo KL. Optimal control of tumor size used to maximize survival time when cells are resistant to chemotherapy. Math Biosci 1992; 110: 201-219.

20. Pereira FL, Pedreira CE, de Sousa JB. A new optimization based approach to experimental combination chemotherapy. Front Med Biol Eng. 1995; 6: 257-268.

21. d'Onofrio A, Ledzewicz U, Maurer $H$, Schattler $H$. On optimal delivery of combination therapy for tumors. Math Biosci. 2009; 222: 13-26.

22. Tan KC, Khor EF, Cai J, Heng CM, Lee TH. Automating the drug scheduling of cancer chemotherapy via evolutionary computation. Artif Intell Med. 2002; 25 : 169-185.

23. Floares A, Floares C, Cucu M, Lazar L. Adaptive neural networks control of drug dosage regimens in cancer chemotherapy. Proc Int Jt Conf Neural Networks. 2003; 1: 154-159.

24. Liang Y, Leung K-S, Mok TSK. A novel evolutionary drug scheduling model in cancer chemotherapy. IEEE Trans Inf Technol Biomed. 2006; 10: 237-245.

25. Petrovski A, McCall J. Multi-objective optimisation of cancer chemotherapy using evolutionary algorithms. Lect Notes Comput Sci. 2001; 1993: 531-545.

26. Alam MS, Algoul S, Hossain MA, Majumder MAA. Chemotherapy Drug Scheduling: A Particle Swarm Optimization Approach. Dhaka Univ J Sci. 2013; 61: 3540.

27. Petrovski A, Sudha B, Mccall J Swarm Optimisation and Genetic Algorithms. 2004; 633-641.

28. Tse S-M, Liang $Y$, Leung $K-S$, Lee K-H, Mok TSK. A memetic algorithm for multiple-drug cancer chemotherapy schedule optimization. IEEE Trans Syst Man Cybern B Cybern; 2007; 84-91.

29. Archana $N$, Vidhyapriya $R$, Benedict $A$, Karthik $C$. Deterministic oscillatory search: a new meta-heuristic optimization algorithm. Sadhana. 2017; 42: 817-826.

30. Algoul S, Alam MS, Hossain MA, Majumder MAA. Multiobjective optimal chemotherapy control model for cancer treatment. Med Biol Eng Comput 2011; 49: 5165.

31. Harrold JM. Model-based design of cancer chemotherapy treatment schedules. Cancer. 2005. 